\title{
Ventilation to Perfusion Ratio
}

National Cancer Institute

\section{Source}

National Cancer Institute. Ventilation to Perfusion Ratio. NCI Thesaurus. Code C94999.

The ratio of activity concentration of a tracer measuring lung air flow per unit volume for a region to that of the activity concentration of a tracer measuring lung blood flow per unit volume for this region. 